\title{
Ginsenoside RgI nanoparticle penetrating the blood-brain barrier to improve the cerebral function of diabetic rats complicated with cerebral infarction
}

\author{
This article was published in the following Dove Press journal: \\ International Journal of Nanomedicine \\ 5 September 2017 \\ Number of times this article has been viewed
}

Junyi Shen

Zhiming Zhao

Wei Shang

Chunli Liu

Beibei Zhang

Lingjie Zhao

Hui Cai

Department of Integrated Traditional and Western Medicine, Jinling Hospital, School of Medicine, Nanjing University, Nanjing, China
Correspondence: Hui Cai Department of Integrated Traditional and Western Medicine, Jinling Hospital, School of Medicine, Nanjing University, 305 East Zhong Shan Road, Nanjing 210002, China

Email njzy_caihui@163.com

\begin{abstract}
Diabetic cerebral infarction is with poorer prognosis and high rates of mortality. Ginsenoside $\operatorname{Rg} 1$ (Rg1) has a wide variety of therapeutic values for central nervous system (CNS) diseases for the neuron protective effects. However, the blood-brain barrier (BBB) restricts $\mathrm{Rg} 1$ in reaching the CNS. In this study, we investigated the therapeutic effects of $\operatorname{Rg} 1$ nanoparticle (PHRO, fabricated with $\gamma$-PGA, L-PAE (H), Rg1, and OX26 antibody), targeting transferrin receptor, on the diabetes rats complicated with diabetic cerebral infarction in vitro and in vivo. Dynamic light scattering analysis shows the average particle size of PHRO was $79 \pm 18 \mathrm{~nm}$ and the polydispersity index $=0.18$. The transmission electron microscope images showed that all NPs were spherical in shape with diameters of $89 \pm 23 \mathrm{~nm}$. PHRO released $\mathrm{Rg} 1$ with sustained release manner and could promote the migration of cerebrovascular endothelial cells and tube formation and even penetrated the BBB in vitro. $\mathrm{PHRO}$ could penetrate the BBB with high concentration in brain tissue to reduce the cerebral infarction volume and promote neuronal recovery in vivo. PHRO was promising to be a clinical treatment of diabetes mellitus with cerebral infarction.
\end{abstract}

Keywords: poly- $\boldsymbol{\gamma}$-glutamic acid, ginsenoside Rg1, OX26, blood-brain barrier

\section{Introduction}

Diabetes has long been related with peripheral nerve degeneration and vascular dysfunction and even injures the central nervous system (CNS) directly. Diabetic cerebral infarction brought about poor functional outcomes and prognoses and high rates of morbidity and mortality after stroke. ${ }^{1}$ Ginseng, the root of Panax ginseng, has been used in several Asian countries for over 2000 years in traditional Chinese medicine. Ginsenoside Rg1 (Rg1) is one of the major components in Panax notoginseng saponins. A number of clinical reports and experiments have indicated that $\mathrm{Rg} 1$ has a variety of therapeutic values: $\mathrm{Rg} 1$ could inhibit lipolysis and promote glucose transporter in adipocytes, improve insulin resistance and abnormal glucose metabolism of organisms, ${ }^{2}$ and improve the neuron function for some CNS disease. ${ }^{3,4}$ Current treatment on diabetic cerebral infarction is mainly focused on reversing neuron degeneration. Many research studies have been focused on protective effect of Rg1 in neurons. However, previous studies demonstrated that Rg1 was poorly absorbed and eliminated quickly ${ }^{5}$ and could not efficiently transport through the blood-brain barrier (BBB) to achieve the effective concentration in the brain tissues. ${ }^{6}$ The BBB was formed 
by brain micro vascular endothelial cells with tight junction to protect the brain from being harmed by the substances of blood. ${ }^{7-9}$ The BBB restricts various therapeutic agents to enter the CNS. ${ }^{9-11}$ According to the general principle, to maximize drug efficacy, the drug should be absorbed and then distributed efficiently to the affected sites. Developing drug delivery strategies across the BBB is important for therapeutic agents to enter the CNS. Achieving more Rg1 penetrate the BBB with the help of the drug delivery system seems to be a promising strategy. Transferrin receptor (TfR), highly concentrated in brain capillary endothelium, could allow transferrin or the antibodies against the TfR to penetrate the BBB via trans-cytosis. ${ }^{12}$ Transferrin or the antibodies against the TfR (OX26 monoclonal antibody) has been used to deliver ${ }^{13}$ liposome-containing digoxin ${ }^{14}$ and nanoparticles (NPs)-containing loperamide ${ }^{15}$ to cross the BBB, which manifested the extraordinary capacity of the receptor-mediated endocytosis and better therapeutic effect. In this study, we developed a poly- $\gamma$-glutamic acid ( $\gamma$-PGA)-based NP to load Rg1 and conjugated with OX26 monoclonal antibody. We investigate the therapeutic effects of the NP on the diabetes rats complicated with diabetic cerebral infarction in vitro and in vivo.

\section{Materials and methods Materials}

Triphenyl tetrazolium chloride (TTC), 1-ethyl-3-(3-dimethyllaminopropyl) carbodiimide hydrochloride (EDCI), L-phenylalanine ethylester (L-PAE), glutaraldehyde, Streptozotocin (STZ) and penicillin-streptomycin sulfate were bought from Sigma-Aldrich Co. (St Louis, MO, USA); 8.0 and $0.4 \mu \mathrm{m}$ transwell chamber was purchased from Corning Incorporated (Corning, NY, USA); Ginsenoside Rg1 was purchased from Shanghai Yuan Ye Biological Technology Co., Ltd; Matrigel was purchased from BD Biosciences (San Jose, CA, USA); rat brain vascular endothelial cells line (RBE4) and endothelial cell medium (ECM) were purchased from ScienCell (Carlsbad, CA, USA); OX26 antibody was purchased from Abcam (Cambridge, UK).

\section{Preparation of PHRO}

We developed an amphiphilic copolymer by modifying the Akaga method. ${ }^{16}$ The copolymer consisted $\gamma$-PGA as the hydrophilic group and L-PAE $(\mathrm{H})$ as hydrophobic side chain. ${ }^{17,18}$ To form the copolymer (PH) of PGA and L-PAE, $3.12 \mathrm{~g}$ L-PAE was conjugated to $2 \mathrm{~g}$ small molecular weight (30 kDa) $\gamma$-PGA by amide reaction under the catalyst EDCI for $24 \mathrm{~h}$ at room temperature. PH-loaded Rg1 could form the
PHR complex. Briefly, PHR was obtained by mixing $5 \mathrm{mg}$ Rg1 with $20 \mathrm{mg}$ PH dissolved in $2 \mathrm{~mL}$ dimethyl sulfoxide, and then the mix was dropped into ultrapure water under a magnetic mixer with a speed of 120 revolutions per minute (rpm). After centrifugation $(12,000 \mathrm{rpm})$ for $10 \mathrm{~min}$, the free $\mathrm{Rg} 1$ was removed and the dry PHR nanoparticles were obtained by a vacuum freeze-drying equipment. Biopolymer samples were stored at $4^{\circ} \mathrm{C}$. TfR targeting NP PHRO was prepared as follows: $10 \mathrm{mg}$ PHR was completely dissolved in ultrapure water under a magnetic mixer with a speed of $120 \mathrm{rpm}$, after that, $4 \mathrm{mg}$ EDCI was added to activate carboxyl residue, followed by the addition of OX26 (1 mL, $100 \mu \mathrm{g} / \mathrm{mL})$ antibody. The reaction was kept in the dark at room temperature for $8 \mathrm{~h}$, and the unconjugated OX26 was removed by centrifugation $(12,000 \mathrm{rpm})$ for $10 \mathrm{~min}$.

\section{Characterization of the PHRO}

The dynamic light scattering (DLS) particle size analyzer (Malvern Instruments, Malvern, UK) was used to characterize the nanosize, polydispersity index (PDI), and the zeta potential of PHRO. The morphology of the PHRO was characterized by transmission electron microscopy (TEM). PHRO was dropped onto the copper wire and then stained with uranyl acetate solution $(1 \%, w / v)$ for TEM (JEOL, Tokyo, Japan) analysis.

\section{Encapsulation efficacy (EE)}

To detect the EE of Rg1 in PHRO, high-performance liquid chromatography (HPLC; Agilent 1100, Palo Alto, CA, USA) with a reversed-phase Lichrospher ODS C18 column was performed. To release Rg1 from PHRO, $5 \mathrm{mg}$ of PHRO was deposited into $1 \mathrm{~mL}$ of acetone. The solution was evaporated by a rotary evaporator under reduced pressure at $40^{\circ} \mathrm{C}$. The leftover was added with $2 \mathrm{~mL}$ of ethanol. The mobile phase was maintained with $0.02 \mathrm{M}$ sodium acetate solution ( $\mathrm{pH} 6.0$ ) and methanol $(80: 20, \mathrm{~V} / \mathrm{V})$, and the flow rate was kept at $1 \mathrm{~mL} / \mathrm{min}$. The wavelength of the detector was maintained at $203 \mathrm{~nm}$ at a temperature of $30^{\circ} \mathrm{C}$. The EE of Rg1 in PHRO was analyzed by the formula $\mathrm{EE}=\mathrm{M} 1 / \mathrm{M} 2$, where $\mathrm{M} 1$ was the quantity of $\mathrm{Rg} 1$ in PHRO and M 2 was the quantity of Rg1 applied in the formula.

\section{Drug release behavior in vitro}

The in vitro release behavior of PHRO was investigated by dialysis method. Briefly, $5 \mathrm{~mL}$ PHRO (Rg1=1 mg/mL) or $\mathrm{Rg} 1$ solution $(1 \mathrm{mg} / \mathrm{mL})$ were added into dialysis bag (molecular weight cutoff, $10 \mathrm{KD}$ ) and then immersed in $50 \mathrm{~mL}$ phosphate-buffered saline (PBS) or normal saline in a 
beaker which was laid on an a shaker at a speed of $150 \mathrm{rpm}$ at $37^{\circ} \mathrm{C}$. At a certain time point, $500 \mu \mathrm{L}$ of samples was obtained from the external dialysis fluid, and the same volume of fresh dialysis fluid was added. The amount of Rg1 released was determined by the HPLC method. Briefly, $20 \mu \mathrm{L}$ samples were injected into HPLC, and the analysis was performed as described in the "Encapsulation efficacy" section.

\section{Diabetic ischemia model}

A total of 120 male Sprague Dawley (SD) rats, weighing $250 \pm 25$ g, were bought from Slac Laboratory Animal (Shanghai, China) and were divided into four groups: 30 rats were randomly selected as control group (group C), 30 rats were selected as diabetic cerebral infarction model group (group M), 30 rats were selected as a diabetic cerebral infarction treated with Rg1 group (group R), and 30 rats were selected as a diabetic cerebral infarction treated with PHRO (group P). Diabetic rat model from groups $\mathrm{M}, \mathrm{R}$, and $\mathrm{P}$ were induced with high-fat feeding for 8 weeks followed by intravenous injection of STZ $50 \mathrm{mg} / \mathrm{kg}$. The control group had only intravenous injection of the same volume of normal saline. The rat tail vein blood glucose values were measured consecutively for 20 days to ensure that diabetic rats were established (blood glucose level $>16.7 \mathrm{mmol} / \mathrm{L}$ ). Longa et $\mathrm{al}^{19}$ described the in vivo model of soft tissue ischemia. The rats were anesthetized with $10 \%$ chloral hydrate $0.35 \mathrm{~mL} / 100 \mathrm{~g}$ through intraperitoneal injection. Neck skin dissection in the middle line was made in the diabetic ischemia model group, Rg1 treatment group, and PHRO treatment group to expose the left common carotid artery, and then one-sided carotid artery ligation was performed. We isolated and ligated the branches of the left external carotid artery under an operating microscope. A 4-0 surgical nylon filament with rounded tip was inserted into the left internal carotid artery through flame. The filament was withdrawn $2 \mathrm{~h}$ after occlusion. A distance of $20 \mathrm{~mm}$ from bifurcation of the common carotid artery to the tip of the suture was maintained. Then, the neck incision was closed to allow rats to recover. The body temperature was carefully supervised during the anesthesia process until the recovery of the rats. After the experiment, the animals were provided free access to food and water. All the studies were performed according to the Guiding Principles for the Care and Use of Laboratory Animals according to the Regulations of the People's Republic of China for Administration of Laboratory Animals. These studies were approved by the Ethics Committee of East China Normal University on Animal Resources.

\section{Animal treatment}

After the diabetic ischemia rat model was established, the Rg1 treatment group and PHRO treatment group were injected with Rg1 $(56.25 \mu \mathrm{M} / \mathrm{kg}$ ) or PHRO (equal to $56.25 \mu \mathrm{M} / \mathrm{kg}$ Rg1) via tail venous. The diabetic ischemia model group and the control group were injected with normal saline at the same time. The treatment lasted for 10 days with a frequency of once a day.

\section{Cerebral vascular endothelial migration Assay}

The migratory activity of cerebral vascular endothelial cell (CEC) line RBE4 was analyzed by a trans-well assay. Briefly, RBE4 cells $\left(5 \times 10^{4}\right)$ were placed in the upper chamber with ECM culture medium with $10 \%$ FBS. The RBE4 cells were incubated with PBS (group C), $10.0 \mu \mathrm{M} \mathrm{Rg1} \mathrm{(group} \mathrm{R),} \mathrm{or}$ PHRO $(\mathrm{Rg} 1=10.0 \mu \mathrm{M}$, group P). After $24 \mathrm{~h}$ of incubation, the filter was washed with PBS and fixed with $4 \%$ paraformaldehyde. Giemsa solution was used to stain the cells for quantification. Three random microscopic fields $(\times 200)$ were counted for cells migration.

\section{Matrigel tube formation assay}

Matrigel $(50 \mu \mathrm{L})$ was placed in $24-w e l l$ plates at $37^{\circ} \mathrm{C}$ for 30 min. RBE4 cells $\left(4 \times 10^{5}\right)$ were grown in fresh ECM with $5 \% \mathrm{FBS}$ and $1 \% \mathrm{P} / \mathrm{S}$ and incubated at $37^{\circ} \mathrm{C}$ for $12 \mathrm{~h}$. RBE4 cells were exposed to PBS (group C), 10.0 $\mu \mathrm{M}$ Rg1 (group R), or PHRO (Rg1=10.0 $\mu \mathrm{M}$, group $\mathrm{P})$. The tube formation amounts of different groups were determined in 10 random fields (magnification $4 \times 10$ ).

\section{Development and validation of in vitro BBB model}

RBE4 cells $\left(4 \times 10^{5}\right)$ were grown in the upper chamber of 24-well trans-well chamber with fresh ECM with 10\% FBS and $1 \% \mathrm{P} / \mathrm{S}$ and incubated at $37^{\circ} \mathrm{C}$ for $24 \mathrm{~h}$. To evaluate the penetrating ability of $\mathrm{PHRO}$ across the $\mathrm{BBB}$, the solution of PBS, free Rg1, and PHRO mixed with ECM with 10\% FBS and $1 \% \mathrm{P} / \mathrm{S}$ were added to the upper chamber of trans-wells $(0.4 \mu \mathrm{m})$. The concentration of free Rg1 and Rg1 in PHRO was $100 \mu \mathrm{M}$. After treatment, $500 \mu \mathrm{L}$ culture medium was obtained from the lower chamber at 20,60, and $180 \mathrm{~min}$. The concentration of Rg1 was measured by HPLC, which was performed as described earlier. After the sample was obtained, a volume of $500 \mu \mathrm{L}$ of ECM culture medium was added to the lower chamber. The penetrating ratio of $\mathrm{Rg} 1$ was then computed by the equation: $\mathrm{ratio} \%=(\mathrm{Cn} / \mathrm{C}$ total) $\times 100 \%$, Cn was the concentration of $\operatorname{Rg} 1$ in the lower 
chamber and $\mathrm{C}$ total was the initial concentration of $\mathrm{Rg} 1$ in the upper chamber.

\section{PHRO permeability in diabetic ischemia rats}

After the establishment of diabetic cerebral infarction model for $24 \mathrm{~h}$, the solution of PBS, Rg1 $(56.25 \mu \mathrm{M} / \mathrm{kg})$ and PHRO $(\mathrm{Rg} 1=56.25 \mu \mathrm{M} / \mathrm{kg}$ ) were given by caudal vein injection to the rats of different groups $(n=10)$ to test the permeability. After administration, the rats were sacrificed by cervical dislocation at the given time intervals (20,60, and $480 \mathrm{~min})$. One milligram brain samples were homogenate with $5 \mathrm{~mL}$ buffer composing distilled water and methanol (1:1 volume). After centrifugation at $12,000 \mathrm{rpm}$ for $10 \mathrm{~min}$, the supernatants were kept at $-80^{\circ} \mathrm{C}$ for HPLC analysis, which was performed as described earlier.

\section{Evaluation of infarct volume by TTC staining}

After the establishment of diabetic cerebral infarction model for 3 days, the rats of different groups were anaesthetized, and the brains were rapidly obtained and cut into $1-\mathrm{mm}$ thick slices and then immersed into $2 \%$ TTC solution at $37^{\circ} \mathrm{C}$ for $30 \mathrm{~min}$. The ischemic damage area of the brain slice was photographed and analyzed by using Photoshop software. ${ }^{20}$ The area without TTC staining was considered as core. ${ }^{21}$ Pink brain tissue was penumbra positioned between core, viable brain tissue was stained with red color, and brain infarction tissue was pale colored. ${ }^{22}$

\section{Histochemistry}

At the end of the experiment, the rats of different groups were treated with $150 \mathrm{~mL}$ of normal saline for intracardiac perfusion, and then they were fixed by perfusion with $200 \mathrm{~mL}$ of $4 \%$ paraformaldehyde. The cerebral hemispheres were cut in the coronal plane, and the brain tissues of different groups were acquired from the identified section of ischemic cerebral cortex and paraffin embedded. ${ }^{23}$ Sections of $5 \mu \mathrm{m}$ were stained with hematoxylin and eosin (HE) and examined by light microscopy (DM2000; Leica Camera, Wetzlar, Germany).

\section{Electron microscopy}

At the end of the experiment, the rats of different groups were treated with intracardiac perfusion and fixation as described in the "Histochemistry" section to fix the brain tissue as soon as possible, and the brain tissues of different groups were acquired from the identified ischemic core and cut into $2 \mathrm{~mm}$ cubes. The samples were fixed in $2.5 \%$ glutaraldehyde (Sigma-Aldrich Co.) for 2 days. The samples were post fixed in $1 \%$ osmium tetroxide, dehydrated, and embedded in Durcupan resin (Fluka, Switzerland). Ultramicrocuts were prepared by using a Reichert Ultra Cut ultra-microtome. The ultramicrocuts were investigated by using a Libra 120 (Carl Zeiss Meditec AG, Jena, Germany) electron microscope.

\section{Statistical analysis}

Statistical analyses were performed by using GraphPad Prism 5 software. One-way analysis of variance test followed by a Bonferroni-corrected posttest were used for the comparisons of more than two groups. A $P<0.05$ was considered statistically significant. All data are presented as means \pm SD.

\section{Results and discussion \\ The size characterization and zeta potential of PHRO}

The particle size and morphology of PHRO was measured by DLS and TEM, respectively. The average particle size of PHRO was $79 \pm 18 \mathrm{~nm}$ (Figure 1A) with a narrow size

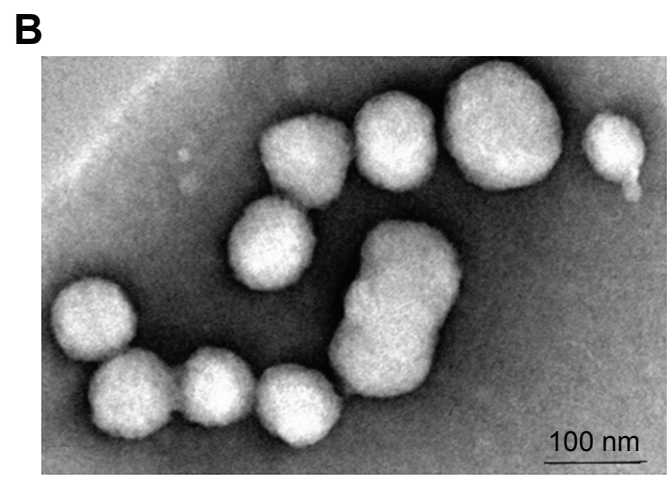

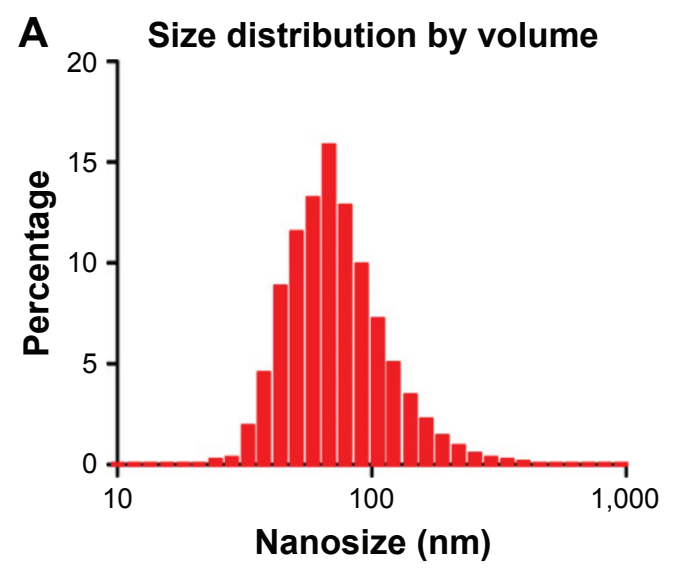

Figure I The size and morphology characterization of PHRO. (A) The nanosize of PHRO was detected by the DLS particle size analyzer. (B) PHRO was dropped onto the copper wire and then stained with uranyl acetate solution for morphology characterized by TEM, Magnification $\times 30,000$.

Abbreviations: DLS, dynamic light scattering; TEM, transmission electron microscope. 
distribution (PDI =0.18). PDI is used to describe the molecular weight distribution of polymers, and smaller PDI implies that the molecular weight distribution is uniform. In general, PDI $<0.3$ was considered as good polymer dispersion. The TEM images showed that all NPs were spherical in shape with an average diameter of $89 \pm 23 \mathrm{~nm}$ (Figure 1B). The particle size was in the scope of nanomedicine. ${ }^{24}$ Zeta potential can be regarded as a stability index of a dispersion system. Stronger potential makes the particles repel each other which can create a more stable dispersion system. ${ }^{25}$ In aqueous phase, the border between the stable and unstable system is generally regarded at either +30 or $-30 \mathrm{mV} .{ }^{26}$ The zeta potential of PHRO was $38 \mathrm{mV}$, which suggested that PHRO could maintain stability in aqueous phase for a strong mutual electrostatic repulsion. In addition, the Rg1 EE was detected by HPLC, and the EE of Rg1 in PHRO was 59.6\%. The high EE of PHRO could prevent the poor aqueous solubility. ${ }^{27}$

\section{In vitro sustained release profile}

In our study, the half-maximal release time $\left(\mathrm{t}_{1 / 2}\right)$ of free $\mathrm{Rg} 1$ was $1.5 \mathrm{~h}$ but that of PHRO was about $29 \mathrm{~h}$. About $81 \%$ of $\mathrm{Rg} 1$ was released, while only $35 \%$ of $\mathrm{Rg} 1$ was released from PHRO at 6 h. At 120 h, 85\% of Rg1 was released from PHRO (Figure 2). The results suggested that the in vitro release profile of Rg1 was a burst release, while Rg1 was released from PHRO in a sustainable manner. The release of drugs from a drug carrier system should be in a sustainable rather than rapid release manner. Compared with rapid release formulation, the slow-release formulation can maintain a long effective concentration of medicine in blood and reduce the drug dose frequency. Free Rg1 in PHRO was released much more slower compared with free Rg1. Hydrophobic drug Rg1

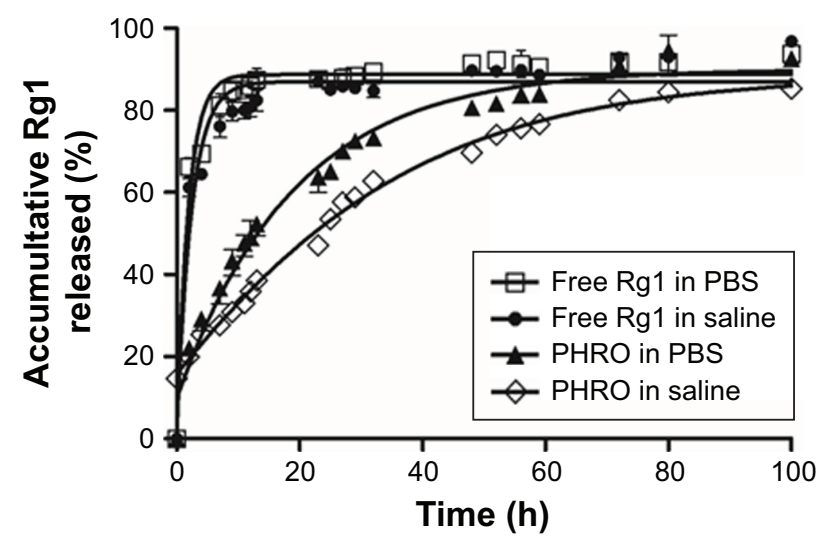

Figure 2 The control released manner of RgI from PHRO. $5 \mathrm{~mL}$ PHRO (I mg/mL) or $\mathrm{Rg} \mid$ solution $(1 \mathrm{mg} / \mathrm{mL})$ were added into a dialysis bag and then immersed in $50 \mathrm{~mL}$ PBS and normal saline in a beaker, which was laid on an a shaker with a speed of $150 \mathrm{rpm}$ at $37^{\circ} \mathrm{C}$ and at given time interval the external dialysis solution was acquired for HPLC analysis.

Abbreviations: HPLC, high-performance liquid chromatography; PBS, phosphatebuffered saline. could be loaded into PHRO which could avoid degradation and increase drug stability.

\section{PHRO facilitated CEC migration}

CEC migratory activity was analyzed by trans-well assay. When RBE4 cells were treated with free $10 \mu \mathrm{M}$ Rg1, the migratory activity was enhanced (Figure $3 \mathrm{~A}$ and $\mathrm{C}$ ) than the group C. When RBE4 was treated with PHRO, the migration ability was stronger than free $\mathrm{Rg} 1$ treatment group. After acute ischemic cerebral infarction, the angiogenesis is accompanied with neuroprotection and neurogenesis. ${ }^{28,29}$ Massive evidences demonstrate that diabetes ${ }^{30,31}$ reduced the level of CECs and the migration ability of CECs. The migration capacity of CECs in patients with diabetes mellitus is impaired in response to ischemia, ${ }^{32,33}$ and it has been speculated that diabetic vascular complications is associated with CEC defects, and the mobilization of CECs is impaired. ${ }^{34}$ Free $\mathrm{Rg} 1$ could improve the migration ability of CECs, and PHRO induced a stronger CEC migration ability compared with free Rg1 which might be due to TfR-mediated endocytosis and more Rg1 entered into the CECs.

\section{PHRO accelerated RBE4 tube formation}

In vitro CEC tube formation ability assay was investigated by the treatment with Rg1 or PHRO. CECs treated with PHRO exhibited more obvious tube formation ability than control group and Rg1 treatment group (Figure 3B and D). Angiogenesis is a good treatment strategy for diabetic cerebral infarction patients. Although Rg1 cannot be easily transported to the neurons, it can exert its protective effect on CNS disease indirectly by improving the tube formation ability of CECs. PHRO exhibited more obvious tube formation ability which was due to the receptor-mediated endocytosis. The regeneration of blood vessels could provide nutritive blood flow and create a microenvironment for neural regeneration and survival. The angiogenesis facilitated neuron protection and neurogenesis. Therefore, PHRO stimulated the angiogenesis potential of CECs which provided a novel therapeutic strategy for the recovery of diabetic cerebral infarction patients.

\section{PHRO across BBB in vitro}

The BBB provides the major CNS protection by hindering the agents from entering the brain. Currently, a major part of the diagnostic and therapeutic delivery systems has been focused on its distribution in brain. In this experiment, the distribution of Rg1 and PHRO was performed by trans-well assay. To evaluate the transport ratio of PHRO across the $\mathrm{BBB}$, varying drug formulations were added to the upper 
A

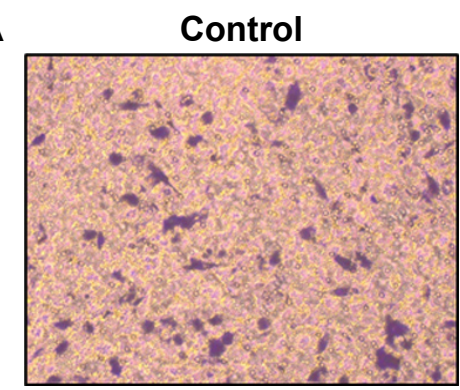

B

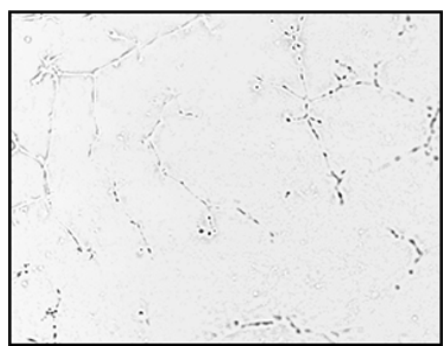

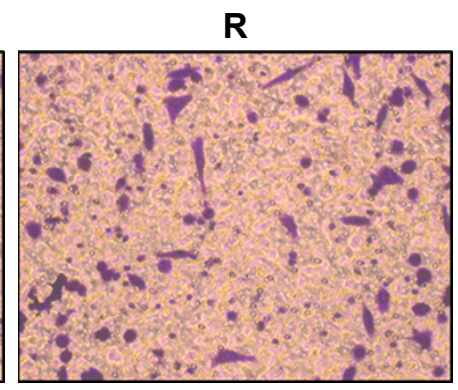
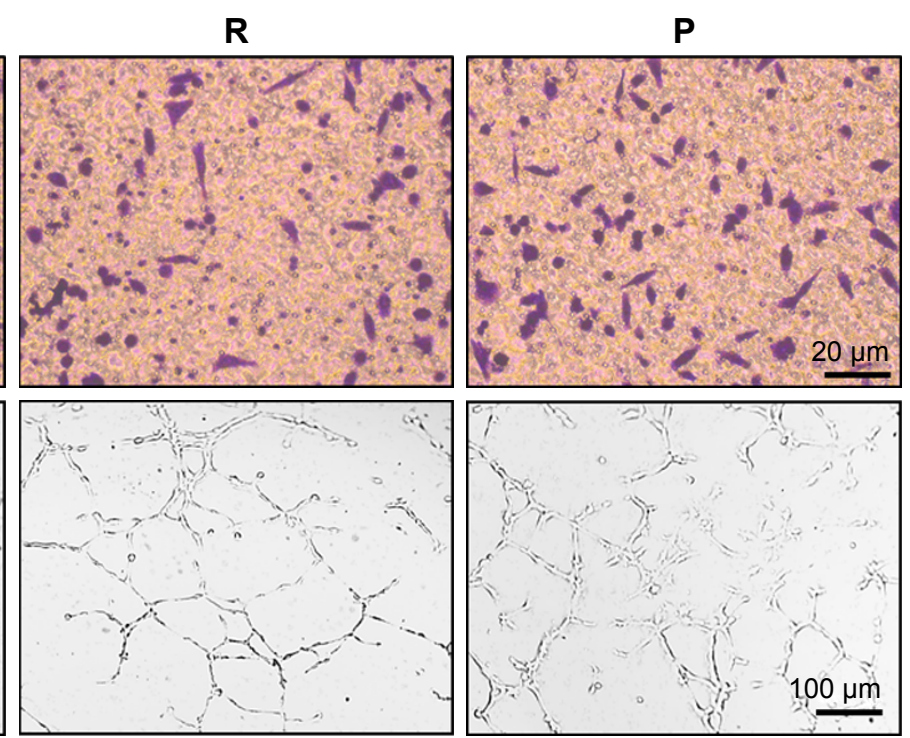

C

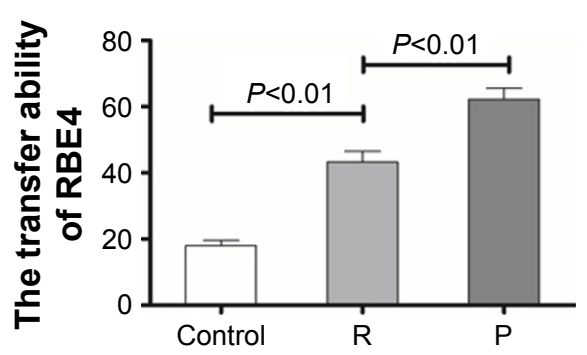

D

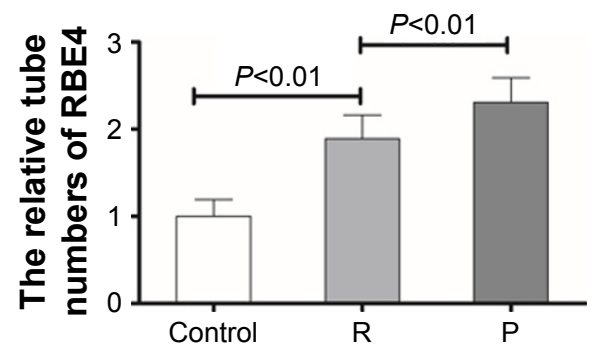

Figure 3 The migration and tube formation of CECs. (A) CEC migration: CECs were incubated with PBS, $10.0 \mu \mathrm{mol}$ RgI and PHRO (RgI=10.0 $\mu$ mol) for $24 \mathrm{~h}$ and detected with 20 -fold objective lens. Magnification $\times 200$. (B) Tube formation of CECs: CECs were incubated with PBS, I0.0 $\mu \mathrm{mol} R \mathrm{Rg}$ and PHRO (RgI=I0.0 $\mu \mathrm{mol}$ ) for $8 \mathrm{~h}$ and detected with 4-fold objective lens. Magnification $\times 40$. (C) Transfer ability of RBE4. (D) The relative tube number of RBE4.

Abbreviations: PBS, phosphate-buffered saline; CECs, cerebrovascular endothelial cells.

chamber of trans-wells $(0.4 \mu \mathrm{m})$, including PBS, free Rg1, and PHRO. The PBS-treated group was evaluated to correct the background signal. It was detected that there was $3.18 \%$ free $\mathrm{Rg} 1$ penetrating the BBB in the lower chamber (Table 1). However, through PHRO treatment, $28.8 \%$ of $\mathrm{Rg} 1$ was detected in the lower chamber (Table 1). Given the results, we discovered that Rg1 was highly distributed in the lower chamber in PHRO group which suggested that PHRO could pass through the BBB in vitro.

\section{PHRO across $B B B$ in vivo}

The objective of the research was to explore the BBB penetrating ability of PHRO. The diabetic ischemia model group

Table I PHRO penetrated blood-brain barrier in vitro

\begin{tabular}{lll}
\hline $\begin{array}{l}\text { Exposure } \\
\text { time }(\mathbf{m i n})\end{array}$ & \multicolumn{2}{l}{ Rg I penetrating rate (\%) } \\
\cline { 2 - 3 } & Free RgI & PHRO \\
\hline 20 & 1.34 & $5.39 *$ \\
60 & 2.89 & $19.48^{*}$ \\
180 & 3.18 & $28.80^{*}$ \\
\hline
\end{tabular}

Notes: PHRO compared with free RgI treatment group, ${ }^{* P}<0.05$. was treated with PBS as the baseline. The Rg1 treatment group showed poor intracerebral distribution. There was much higher $\operatorname{Rg} 1$ accumulation $(259.43 \pm 72.33 \mathrm{ng} / \mathrm{mL})$ in rat brain compared with free $\operatorname{Rg} 1(19.18 \pm 9.39 \mathrm{ng} / \mathrm{mL})$ treatment (Table 2), which indicated that PHRO could effectively penetrate BBB. PHRO NP could maintain longer Rg1 effect time and realized higher enrichment in brain tissue that was beneficial for the recovery of cerebral infarction. This evidence was important for understanding the mechanism of some CNS-targeted drugs, which possess neuron protective effects while having very limited brain distribution. ${ }^{8,35,36}$ The main obstacle in diabetic cerebral infarction treatment research is the BBB, which greatly blocks the delivery of

Table 2 PHRO penetrated blood-brain barrier in vivo

\begin{tabular}{lll}
\hline $\begin{array}{l}\text { Exposure } \\
\text { time }(\mathbf{m i n})\end{array}$ & \multicolumn{2}{l}{ Brain Rg I concentration $(\mathbf{n g} / \mathbf{m L})$} \\
\cline { 2 - 3 } & Free Rg I & PHRO \\
\hline 20 & $8.34 \pm 2.34$ & $55.84 \pm 12.63^{*}$ \\
60 & $18.89 \pm 4.55$ & $119.85 \pm 36.89 *$ \\
480 & $19.18 \pm 9.39$ & $259.43 \pm 72.33^{*}$ \\
\hline
\end{tabular}

Notes: PHRO treatment group compared with free RgI treatment group, ${ }^{* P}<0.05$. 

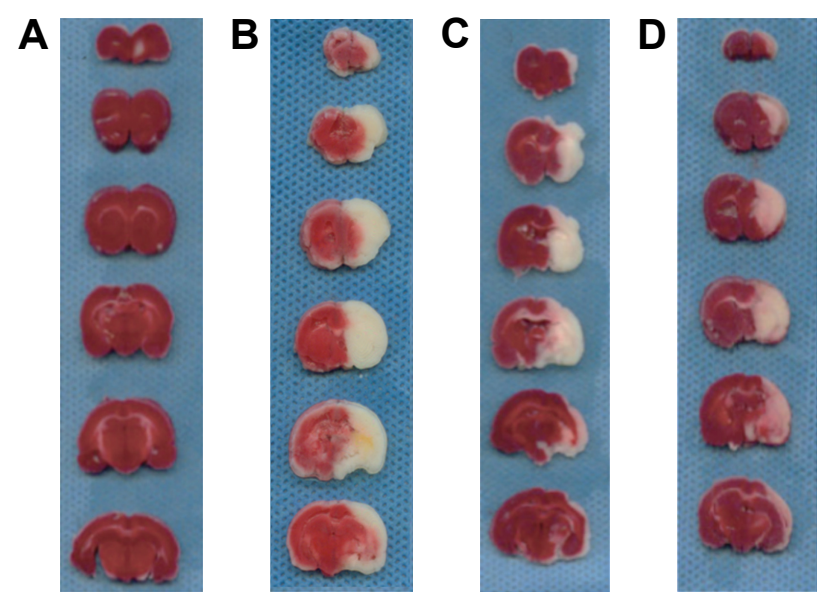

Figure 4 Brain TTC staining. Brains were sliced into I-mm thick slices and then immersed into $2 \%$ TTC solution at $37^{\circ} \mathrm{C}$ for $30 \mathrm{~min}$. Brain infarction was stained with pale-colored and the normal tissues were stained with red color. The ischemic damage area of the brain slice was photographed and analyzed by using Photoshop. (A) Control group rats treated with PBS. (B) Diabetic cerebral infarction rat model group treated with PBS. (C) Diabetic cerebral infarction rat model group treated with $\mathrm{RgI}(56.25 \mu \mathrm{M} / \mathrm{kg})$. (D) Diabetic cerebral infarction rat model group treated with PHRO $(\mathrm{RgI}=56.25 \mu \mathrm{M} / \mathrm{kg})$.

Abbreviations: TTC, triphenyl tetrazolium chloride; PBS, phosphate-buffered saline.

drugs to brain tissues. As such, the BBB causes a great deal of difficulty in the treatment of CNS diseases. According to reports, OX26 antibody could help transport drugs across the BBB and finally increase the amount of drugs in the brain because of high expression of TfR on the BBB. OX26 is able to be specifically banded with TfR and enters cells through endocytosis. Thus, in this research, OX26 was bound to the surface of the TfR as a targeting ligand, which played an important role in contributing to the PHRO to cross the BBB and improve the brain targeting ability.

\section{Brain tissue TTC staining}

The infarction volumes were evaluated by TTC staining. TTC method is often used to measure the area of cerebral infarction. The normal tissue is red and the cerebral infarction area is white. Gross specimens stained with TTC were illustrated in Figure 4. Rat brains from the four groups were subjected to morphometric study to detect the infarcted area. There was swelling of the right hemisphere in $\mathrm{M}, \mathrm{R}$, and $\mathrm{P}$ groups. The infarct volume of the cortical infarct measured in the animals was $264.02 \pm 10.07 \mathrm{~mm}^{3}$ in $\mathrm{M}$ group, $198.72 \pm 9.29 \mathrm{~mm}^{3}$ in $\mathrm{Rg} 1$ treatment group, and $126 \pm 16.49 \mathrm{~mm}^{3}$ in PHRO treatment group (Table 3).
There was no brain tissue necrosis in the control group. There was obvious necrosis in diabetic ischemia model group and Rg1 treatment group, the necrosis of the PHRO treatment group was reduced than that of the diabetic ischemia model group and Rg1 treatment group. After the diabetic cerebral infarction, ischemia first appeared in the level of microcirculation. Then, various injury mechanisms were initiated and leaded to neuronal injury. Timely and effective treatment on impaired nerve tissue with therapeutic agents is important for the recovery. However, the BBB hinders drug entry into the brain. Therefore, it is of great significance for drugs to penetrate the BBB. The therapeutic effect of diabetes mellitus cerebral infarction from PHRO was superior to that of free Rg1 treatment which mainly accounted for the PHRO BBB penetrating ability.

\section{HE histochemistry}

In the control group, the cells were intact in structure. The cell nuclei ranged orderly with distinct blue staining and the cytoplasm was without necrosis or degeneration (Figure 5A). In diabetic ischemia model group, cell nuclei exhibited pyknosis and the cytoplasm staining was uneven with slight damage (Figure 5B). In Rg1 treatment group, the cells were with irregular arrangement and interstitial edema. The nucleus exhibited pyknosis and rupture, part of the nuclei vanished, and the morphological structure was fuzzy (Figure 5C). Compared to the $\mathrm{Rg} 1$ treatment group, the cellular structures of group PHRO treatment group were clear, cells arranged orderly, nuclei dispersion phenomenon was slightly weakened, and the number of cells swollen was reduced (Figure 5D). PHRO penetrated BBB via receptor-mediated endocytosis and brought more $\operatorname{Rg} 1$ for brain nerve tissue which exhibited better $\mathrm{Rg} 1$ treatment effect for the CNS.

\section{TEM}

In the control group (Figure 6A), the capillary (Cap) walls were with tight junction and the neurons were clear with double membrane; mitochondria were rich; structure of endoplasmic reticulum was intact; the myelin sheath (MS) fibers were regular in structure; axons were clear; chromatin was arranged orderly with a high electronic permeability. In diabetic ischemia model group (Figure 6B), the

Table 3 TTC staining of cerebral infarct volume of different groups $(n=10)$

\begin{tabular}{lllll}
\hline Groups & Control & M & R & P \\
\hline Infarct volume & $0 \mathrm{~m}^{3}$ & $264.02 \pm 10.07 \mathrm{~mm}^{3}$ & $198.72 \pm 9.29 \mathrm{~mm}^{3, *}$ & $126.32 \pm 16.49 \mathrm{~mm}^{3, * . \#}$ \\
\hline
\end{tabular}

Notes: $\mathrm{RgI}$ treatment group or PHRO treatment group compared with diabetic ischemia model group, $* \mathrm{P}<0.05$. PHRO treatment group compared with RgI treatment group, ${ }^{\#} P<0.05$. 

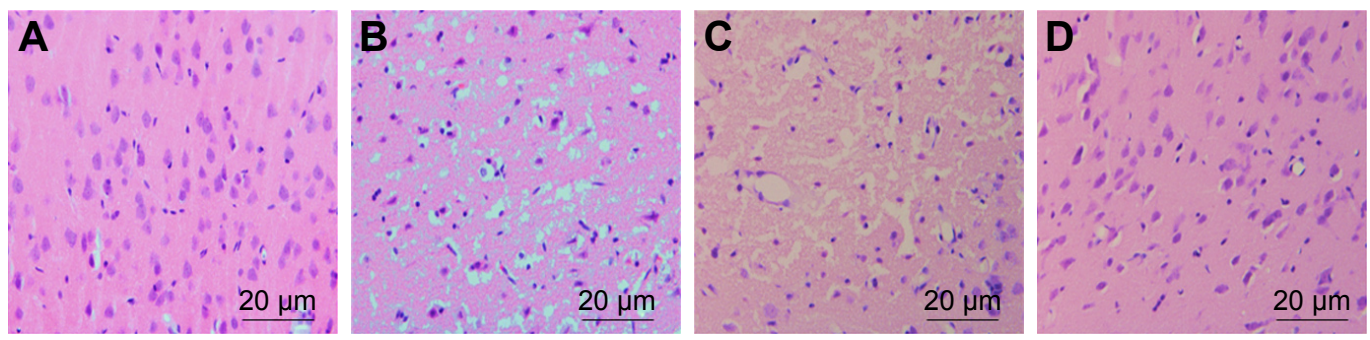

Figure 5 Histochemistry of cerebral infarction tissue. The cerebral hemispheres were cut in the coronal plane, the brain tissues were acquired from the identified ischemic core and paraffin embedded. Sections of $5 \mu \mathrm{m}$ were stained with HE solution. (A) Control group treated with PBS. (B) Diabetic cerebral infarction rat model group treated with PBS. (C) Diabetic cerebral infarction rat model group treated with RgI. (D) Diabetic cerebral infarction rat model group treated with PHRO. Magnification $\times 200$.

Abbreviations: HE, hematoxylin and eosin; PBS, phosphate-buffered saline.

diameter of Cap increased, the Cap walls were relatively thin and the cell junctions were not tight; MS was unclear or merging; and axons vanished. In the Rg1 treatment group (Figure 6C), the Cap walls were thicker than that of the diabetic ischemia model group and the neuron morphology was atypically condensed; organelles were broken or gone; MS was unclear or with demyelination; mitochondria were destroyed. Compared to the Rg1 treatment group R,
A

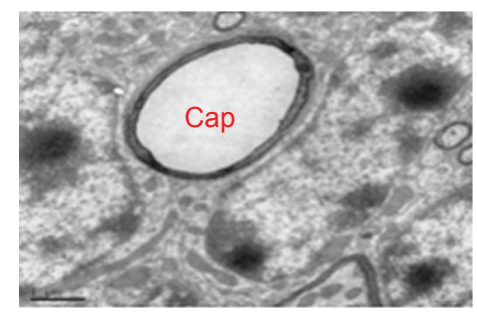

B

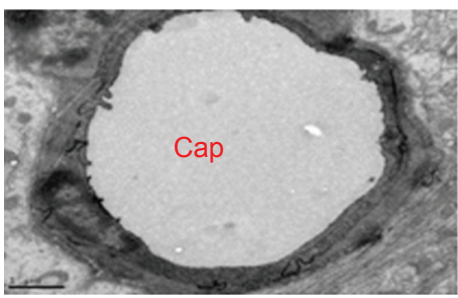

C

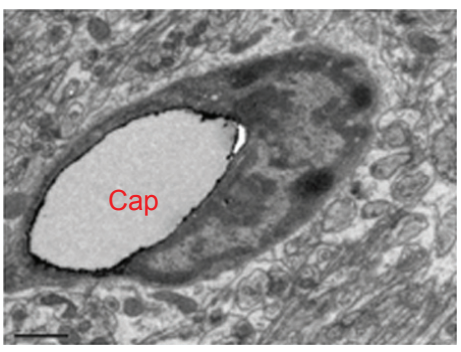

D

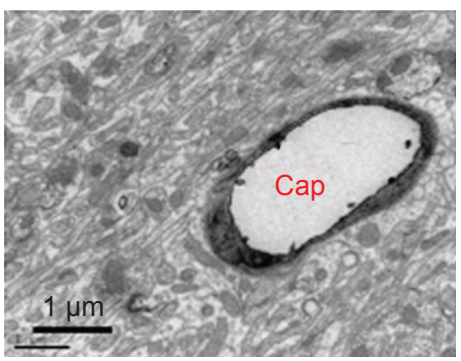

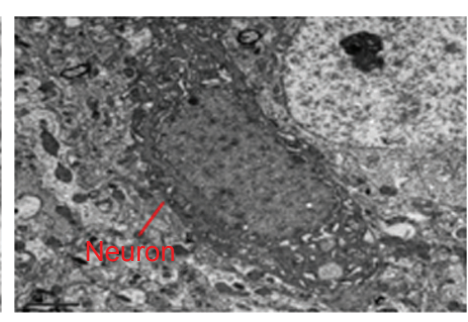
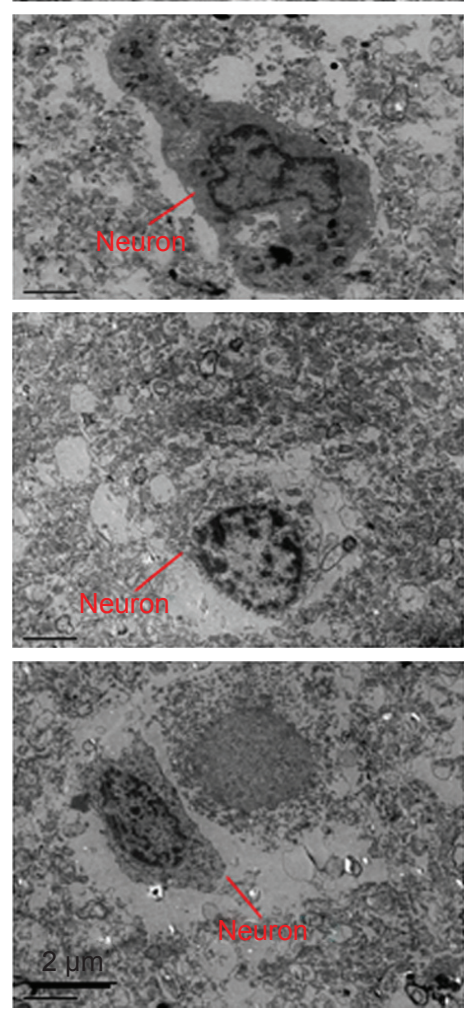
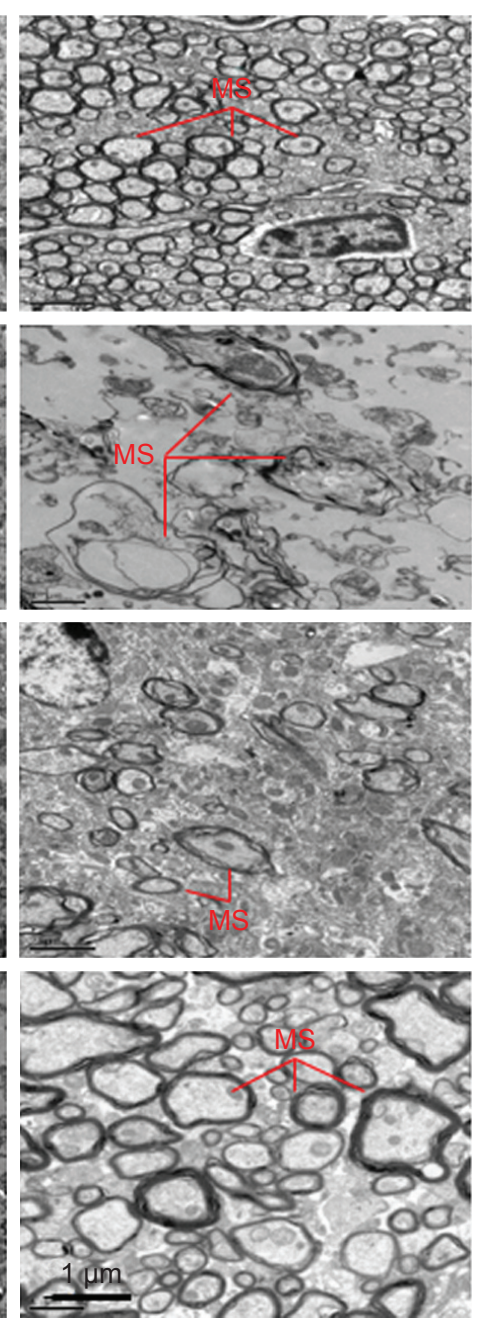

Figure 6 Cerebral infarction tissue detected by TEM. The rats were processed with cardiac perfusion and fixation, and the brain tissues were acquired from the identified ischemic core and fixed with glutaraldehyde and post fixed in osmium tetroxide, dehydrated, and embedded in Durcupan resin. Ultramicrocuts were made for TEM detection. (A) Control group treated with PBS. (B) Diabetic cerebral infarction rat model group treated with PBS. (C) Diabetic cerebral infarction rat model group treated with RgI (56.25 $\mu$ M/kg). (D) Diabetic cerebral infarction rat model group treated with PHRO (RgI=56.25 $\mu \mathrm{M} / \mathrm{kg}$ ). Magnification for Cap and MS was $\times 10,000$, magnification for Neuron was $\times 20,000$. Abbreviations: TEM, transmission electron microscopy; Cap, capillaries; MS, myelin sheath; PBS, phosphate-buffered saline. 
the Cap wall junctions were much tighter; the nerve damage improved significantly in the PHRO treatment group (Figure 6D), the quantity of mitochondria increased, and the chromatin aggregated. The phenomena of loss of MS and myelinoclasis were improved. Compared to the $\mathrm{Rg} 1$ treatment group, after PHRO treatment, myelin fiber injury improved distinctly and the MS and near axon became obvious. The angiogenesis abilities of CECs from diabetic patients and animal models are impaired, and the number of CECs is lower than the normal glycemic conditions. In the PHRO treatment group, the improvement of microstructure of Cap walls and neurons in cerebral infarction areas were contributed to the PHRO BBB penetrating ability which was through receptor-mediated endocytosis. The TfR-targeted drug delivery method exhibited better therapeutic effects of Rg1 for cerebral infarction treatment.

\section{Conclusion}

The purpose of the research was to investigate whether PHRO could cross the BBB to protect the nerve tissue. The major findings of this work included: 1) DLS and TEM showed that the NPs were spherical in shape with narrow nanosize distribution and with sustained release manner. 2) The in vitro experiment revealed that $\mathrm{PHRO}$ enhanced CEC migration and CEC tube formation and could cross the BBB. 3) The in vivo experiments exhibited that $\mathrm{PHRO}$ could increase $\mathrm{Rg} 1$ brain distribution in the diabetic cerebral infarction treatment group. The HE staining and TEM results revealed that brain necrosis of the PHRO group was improved than that of the free $\mathrm{Rg} 1$ treatment group.

\section{Acknowledgment}

The research was supported by grants of Natural Science Foundation of China (81273725).

\section{Disclosure}

The authors report no conflicts of interest in this work.

\section{References}

1. Biller J, Love BB. Diabetes and stroke. Medical Clin North Am. 1993; 77(1):95-110.

2. Shang WB, Yu XZ, Wang GQ, Zhao J. [Effect of ginsenoside Rb1 in ameliorating insulin resistance and ectopic fat deposition in obese mice induced by high fat diet]. Zhongguo Zhong Yao Za Zhi. 2013; 38(23):4119-4123. Chinese.

3. Wu Y, Pan X, Xu Y, et al. Optimization of combinations of ginsenoside$\mathrm{Rg} 1$, ginsenoside-Rb1, evodiamine and rutaecarpine for effective therapy of mouse migraine. J Nat Med. 2016;70(2):207-216.

4. Zhou T, Zu G, Zhang X, et al. Neuroprotective effects of ginsenoside $\mathrm{Rg} 1$ through the Wnt/beta-catenin signaling pathway in both in vivo and in vitro models of Parkinson's disease. Neuropharmacology. 2016; 101:480-489.
5. Sun J, Wang G, Haitang X, Hao L, Guoyu P, Tucker I. Simultaneous rapid quantification of ginsenoside $\mathrm{Rg} 1$ and its secondary glycoside Rh1 and aglycone protopanaxatriol in rat plasma by liquid chromatographymass spectrometry after solid-phase extraction. J Pharm Biomed Anal. 2005;38(1):126-132.

6. Zhou Y, Li HQ, Lu L, et al. Ginsenoside Rg1 provides neuroprotection against blood brain barrier disruption and neurological injury in a rat model of cerebral ischemia/reperfusion through downregulation of aquaporin 4 expression. Phytomedicine. 2014;21(7):998-1003.

7. Begley DJ, Brightman MW. Structural and functional aspects of the blood-brain barrier. Prog Drug Res. 2003;61:39-78.

8. Pardridge WM. The blood-brain barrier: bottleneck in brain drug development. NeuroRx. 2005;2(1):3-14.

9. Wolburg H, Lippoldt A. Tight junctions of the blood-brain barrier: development, composition and regulation. Vascul Pharmacol. 2002;38(6): 323-337.

10. Haseloff RF, Dithmer S, Winkler L, Wolburg H, Blasig IE. Transmembrane proteins of the tight junctions at the blood-brain barrier: structural and functional aspects. Semin Cell Dev Biol. 2015;38:16-25.

11. Abbott NJ. Prediction of blood-brain barrier permeation in drug discovery from in vivo, in vitro and in silico models. Drug Discov Today Technol. 2004;1(4):407-416.

12. Chang J, Jallouli Y, Kroubi M, et al. Characterization of endocytosis of transferrin-coated PLGA nanoparticles by the blood-brain barrier. Int J Pharm. 2009;379(2):285-292.

13. Karatas H, Aktas Y, Gursoy-Ozdemir Y, et al. A nanomedicine transports a peptide caspase-3 inhibitor across the blood-brain barrier and provides neuroprotection. J Neurosci. 2009;29(44):13761-13769.

14. Deeken JF, Loscher W. The blood-brain barrier and cancer: transporters, treatment, and Trojan horses. Clin Cancer Res. 2007;13(6):1663-1674.

15. Ulbrich K, Hekmatara T, Herbert E, Kreuter J. Transferrin- and transferrin-receptor-antibody-modified nanoparticles enable drug delivery across the blood-brain barrier (BBB). Eur J Pharm Biopharm. 2009;71(2):251-256.

16. Akagi T, Kaneko T, Kida T, Akashi M. Preparation and characterization of biodegradable nanoparticles based on poly(gamma-glutamic acid) with 1-phenylalanine as a protein carrier. J Control Release. 2005; 108(2-3):226-236.

17. Geng X, Ye H, Feng Z, et al. Synthesis and characterization of cisplatinloaded, EGFR-targeted biopolymer and in vitro evaluation for targeted delivery. J Biomed Mater Res. 2012;100(10):2839-2848.

18. Zhang L, Wang T, Li Q, et al. Fabrication of novel vesicles of triptolide for antirheumatoid activity with reduced toxicity in vitro and in vivo. Int J Nanomedicine. 2016;11:2663-2673.

19. Longa EZ, Weinstein PR, Carlson S, Cummins R. Reversible middle cerebral artery occlusion without craniectomy in rats. Stroke. 1989; 20(1):84-91.

20. Yang Y, Shuaib A, Li Q. Quantification of infarct size on focal cerebral ischemia model of rats using a simple and economical method. J Neurosci Methods. 1998;84(1-2):9-16.

21. Bederson JB, Pitts LH, Germano SM, Nishimura MC, Davis RL, Bartkowski HM. Evaluation of 2,3,5-triphenyltetrazolium chloride as a stain for detection and quantification of experimental cerebral infarction in rats. Stroke. 1986;17(6):1304-1308.

22. Gill R, Sibson NR, Hatfield RH, et al. A comparison of the early development of ischaemic damage following permanent middle cerebral artery occlusion in rats as assessed using magnetic resonance imaging and histology. J Cereb Blood Flow Metab. 1995;15(1):1-11.

23. Zhao L, Xu J, Wang Q, et al. Protective effect of rhGLP-1 (7-36) on brain ischemia/reperfusion damage in diabetic rats. Brain Res. 2015; 30:153-159

24. Arfors KE, Rutili G, Svensjo E. Microvascular transport of macromolecules in normal and inflammatory conditions. Acta Physiol Scand Suppl. 1979;463:93-103.

25. Mishra PR, Al Shaal L, Muller RH, Keck CM. Production and characterization of Hesperetin nanosuspensions for dermal delivery. Int $J$ Pharm. 2009;371(1-2):182-189. 
26. Bhattacharjee S. DLS and zeta potential - what they are and what they are not? J Control Release. 2016;235:337-351.

27. Patil S, Lis LG, Schumacher RJ, et al. Phosphonooxymethyl prodrug of triptolide: synthesis, physicochemical characterization, and efficacy in human colon adenocarcinoma and ovarian cancer xenografts. J Med Chem. 2015;58(23):9334-9344.

28. Thored P, Wood J, Arvidsson A, Cammenga J, Kokaia Z, Lindvall O. Long-term neuroblast migration along blood vessels in an area with transient angiogenesis and increased vascularization after stroke. Stroke. 2007;38(11):3032-3039.

29. Imitola J, Raddassi K, Park KI, et al. Directed migration of neural stem cells to sites of CNS injury by the stromal cell-derived factor 1alpha/ CXC chemokine receptor 4 pathway. Proc Natl Acad Sci U S A. 2004; 101(52):18117-18122.

30. Loomans CJ, de Koning EJ, Staal FJ, et al. Endothelial progenitor cell dysfunction: a novel concept in the pathogenesis of vascular complications of type 1 diabetes. Diabetes. 2004;53(1):195-199.
31. Fadini GP, Miorin M, Facco M, et al. Circulating endothelial progenitor cells are reduced in peripheral vascular complications of type 2 diabetes mellitus. J Am Coll Cardiol. 2005;45(9):1449-1457.

32. Calanna S, Christensen M, Holst JJ, et al. Secretion of glucagon-like peptide- 1 in patients with type 2 diabetes mellitus: systematic review and meta-analyses of clinical studies. Diabetologia. 2013;56(5):965-972.

33. Balestrieri ML, Servillo L, Esposito A, et al. Poor glycaemic control in type 2 diabetes patients reduces endothelial progenitor cell number by influencing SIRT1 signalling via platelet-activating factor receptor activation. Diabetologia. 2013;56(1):162-172.

34. Maiorino MI, Bellastella G, Petrizzo M, et al. Circulating endothelial progenitor cells in type 1 diabetic patients with erectile dysfunction. Endocrine. 2015;49(2):415-421.

35. Teichberg VI. From the liver to the brain across the blood-brain barrier. Proc Natl Acad Sci US A. 2007;104(18):7315-7316.

36. Pardridge WM. Drug and gene delivery to the brain: the vascular route. Neuron. 2002;36(4):555-558.
International Journal of Nanomedicine

\section{Publish your work in this journal}

The International Journal of Nanomedicine is an international, peerreviewed journal focusing on the application of nanotechnology in diagnostics, therapeutics, and drug delivery systems throughout the biomedical field. This journal is indexed on PubMed Central, MedLine, CAS, SciSearch ${ }^{\circledR}$, Current Contents ${ }^{\circledR} /$ Clinical Medicine,

\section{Dovepress}

Journal Citation Reports/Science Edition, EMBase, Scopus and the Elsevier Bibliographic databases. The manuscript management system is completely online and includes a very quick and fair peer-review system, which is all easy to use. Visit http://www.dovepress.com/ testimonials.php to read real quotes from published authors. 